\title{
Preface for the special issue of MTAP following CBMI 2010
}

\author{
Georges Quénot • Jenny Benois-Pineau • \\ Régine André-Obrecht
}

Published online: 11 November 2010

(C) Springer Science+Business Media, LLC 2010

Content-based multimedia Indexing is a large research area covering various aspects of media analysis, description and retrieval. This dynamic research is moving very fast to more and more complex methods based on the latest advances in feature design, feature fusion, semi-supervised learning.

It is 11 years now that the first Workshop on Content Based Multimedia Indexing (CBMI) was organized. Since then CBMI evolved as one of the main forums for the presentation of the latest research advances in the field. It combines academic presentations, system demonstrations, discussions on industrial needs and product developments in multimedia indexing, search, retrieval, navigation and browsing. CBMI is now a wellestablished forum serving the needs to discuss developments in the field, foster relations among fragmented research groups across Europe and improve synergies between key players and research teams in the EU. Large EU cooperative projects and groups from several countries in and outside Europe are involved.

Following the seven successful previous events of CBMI (Toulouse 1999, Brescia 2001, Rennes 2003, Riga 2005, Bordeaux 2007, London 2008 and Chania 2009), the 8th International Workshop on Content-Based Multimedia Indexing (CBMI 2010) was hosted by the Laboratory of Informatics of Grenoble (LIG).

CBMI 2010 received 46 submissions from which the program committee selected 19 as oral presentations and eight as poster presentations. Among these papers, some deserved more space to present ideas and results and a broader diffusion; MTAP offered to publish the best of them. This year, the special issue associated to CBMI has been open to authors that did not presented a paper at the workshop. 31 papers were submitted, seven of which

G. Quénot $(\square)$

LIG-CNRS UMR 5217, BP 53, 38041 Grenoble Cedex 9, France

e-mail: georges.quenot@imag.fr

J. Benois-Pineau

LABRI UMR 5800, UniversitéBordeaux1/Bordeaux2/CNRS/ENSEIRB, Talence Cedex, France

R. André-Obrecht

University Paul Sabatier, Toulouse, France 
were a new and enlarged version of a paper presented at CBMI. All papers were subject to a review according to the journal rules. Eleven among them were finally accepted for publication.

We are deeply grateful to all those who have helped to accomplish the task of successfully organizing CBMI 2010 and this special issue of MTAP. Special thanks to the Program Committee members and the reviewers of both the conference and this issue who have contributed to the high quality of the works presented here, to the Steering Committee members, the special session organizers and the invited speakers who undoubtedly helped to enhance the profile of CBMI 2010 and to attract many high quality papers.

Finally, we are indebted to all the authors of the papers submitted to the workshop and to this special issue. They constitute the true force behind the success of the workshop and are the first accountable for the quality and the interest of this issue.

\title{
Georges Quénot \\ CBMI'10 General Chair
}

\author{
Alan Hanjalic \\ Cees Snoek \\ Régine André-Obrecht \\ CBMI'10 Program Chairs
}

\author{
Georges Quénot \\ Jenny Benois-Pineau \\ Régine André-Obrecht \\ MTAP Guest Editors
}

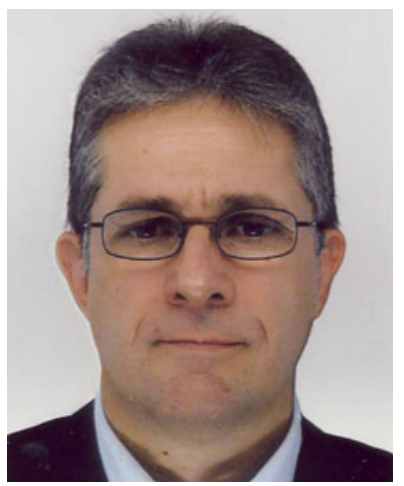

Dr. Georges Quénot is Researcher at CNRS (French National Centre for Scientific Research). He has an engineer diploma of the French Polytechnic School (1983), a PhD in computer science (1988) from the University of Orsay and an Habilitation à Diriger la Recherche in Computer Science (1998) from the University of Orsay. He is the author and co-author of more than 80 papers in international journals, conference proceedings and book chapters. He has served on numerous program committees in international conferences and workshops: ACM MM, ICIP, ICME, CIVR, MMM, CBMI ... He is currently with the Multimedia Information Indexing and Retrieval group (MRIM) of the Laboratoire d'informatique de Grenoble (LIG) where he is responsible for their activities on video indexing and retrieval. His current research activity is about semantic indexing of image and video documents using supervised learning, networks of classifiers and multimodal fusion. 


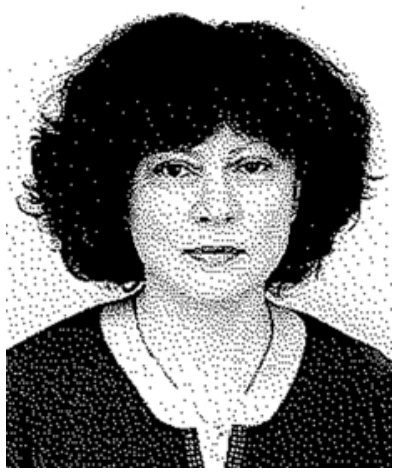

Jenny Benois-Pineau is a full professor of Computer science at the University Bordeaux 1 and chair of Video Analysis and Indexing research group in Image and Sound Department of LABRI UMR 58000 UniversitéBordeaux1/Bordeaux2/CNRS/ENSEIRB. She is also a deputy scientific director of theme B of French national research unity GDR CNRS ISIS.

She obtained her $\mathrm{PhD}$ degree in Signals and Systems in Moscow and her Habilitation à Diriger la Recherche in Computer Science and Image Processing from University of Nantes France.

Her topics of interest include image and video analysis and indexing, motion analysis and content description for content-based multimedia retrieval.

She is the author and co-author of more than 100 papers in international journals, conference proceedings, book chapters. She has tutored and co-tutored $18 \mathrm{PhD}$ students and 19 research masters.

She is associated editor of EURASIP Signal Processing:Image Communication, Elsevier and ACM Multimedia Tools and applications, Springer journals. She has served on numerous program committees in international conferences and workshops: ACM MM, CIVR, CBMI, AMR, IPTA, SAMT, ECMCS...

She has served as expert for European Commission since FP4 and has been a member of Technical Advisory group for Media programme EACEA DG Culture, CE. She is a member of Multimedia Commission of French Ministry of National Education and member of scientific board of International Center for Mathematical Modelling at the University of Växjo, Sweden.

She has been coordinator or leading researcher in international research projects Platon, Balaton, IP XMedia, French representative in COST292 European action, national research projects ANR and numerous projects with French industrial companies.

She gave invited lectures at the universities of Sussex (GB), UPC (Spain), UNAM (Mexico), University of North Carolina at Chapel Hill (USA), Brooklyn Polytechnic (USA), Firenze (Italy). Since 2010 she has been Director of Computer Science Department at the Faculty of Mathematics and Computer Science at the University Bordeaux 1

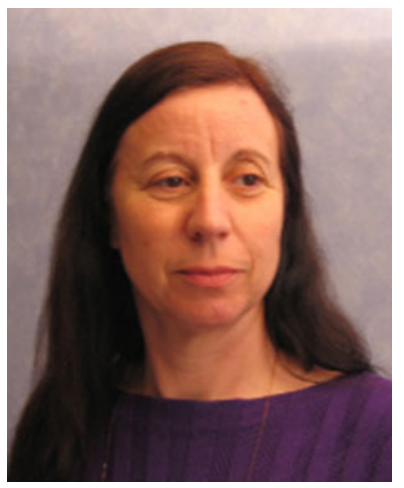

Régine Andre-Obrecht was born in Orléans (France) in 1955. She studied mathematics and probability theory at Ecole Normale Supérieure of Fontenay aux Roses, and she received the diploma of "Agregation de 
Mathematiques" in 1981. In 1982-1985, she worked with the Institut de Recherches en Informatique et Systèmes Aléatoires de Rennes (IRISA) at the department of Signal Processing with Albert Benveniste. She received the doctorat of $3 \mathrm{e}$ cycle from the university of Rennes in 1985. As a researcher at the CNRS (National Center for Scientific Research), between 1986 and 1991, she worked at IRISA, and in 1991 she joined the Institut de Recherches en Informatique de Toulouse (IRIT) of the University Paul Sabatier of Toulouse. She received the diploma "Habilitation à Diriger des Recherches" of the University of Rennes in 1993. She is professor of the University Paul Sabatier since 1999 and since 2008 she is Director of the Computer Science Department of the University Paul Sabatier.

Her research domain belongs to Signal Processing and Pattern Recognition and her privileged approach is the statistical approach. Her main research topics are Speech analysis with a special attention for segmentation and robust analysis as speech recognition preprocessing, Speech, Speaker and Language recognition which includes audio visual recognition, sound duration modeling, Audio content description as speech/music discrimination and jingle detection, and Fusion information. Member of the group SAMoVA "Structuration, Analysis, Modeling of Video and Audio documents", Régine André-Obrecht is implied in several scientific projects (national as ANR projects, European as the NoE Muscle).

She was member of the Direction Committee of the National GDR (Group of Research) "Information, Signal, Image, Vision", and consequently she was a main actor of the "Multimedia Indexing" French community; she co-organized the first CBMI workshop (1999) at Toulouse and she is a member of the steering committee of the CBMI workshop since its founding. Member of the International Speech Communication Association, she was a member of the program committee of Interspeech 2007, RFIA 2008 and CARI 2008, CARI 2010. Invited Editor for special issues of Speech Communication (July 2000), of EURASIP journal on Image and Video Processing (2008), of MultimediaTools Applications (2009), she is a member of the Redaction Committee of the Journal "Traitement de Signal" and the Journal "ARIMA". 